\title{
A bibliometric analysis of the development of next generation active nanotechnologies
}

DOI:

10.1007/s11051-016-3578-8

\section{Document Version}

Accepted author manuscript

Link to publication record in Manchester Research Explorer

\section{Citation for published version (APA):}

Suominen, A., Li, Y., Youtie, J., \& Shapira, P. (2016). A bibliometric analysis of the development of next generation active nanotechnologies. Journal of Nanoparticle Research, 18(9), [270]. https://doi.org/10.1007/s11051-016-35788

\section{Published in:}

Journal of Nanoparticle Research

\section{Citing this paper}

Please note that where the full-text provided on Manchester Research Explorer is the Author Accepted Manuscript or Proof version this may differ from the final Published version. If citing, it is advised that you check and use the publisher's definitive version.

\section{General rights}

Copyright and moral rights for the publications made accessible in the Research Explorer are retained by the authors and/or other copyright owners and it is a condition of accessing publications that users recognise and abide by the legal requirements associated with these rights.

\section{Takedown policy}

If you believe that this document breaches copyright please refer to the University of Manchester's Takedown Procedures [http://man.ac.uk/04Y6Bo] or contact uml.scholarlycommunications@manchester.ac.uk providing relevant details, so we can investigate your claim.

\section{OPEN ACCESS}




\title{
A Bibliometric Analysis of the Development of Next Generation Active Nanotechnologies
}

\author{
Arho Suominen ${ }^{a}$, Yin $\mathrm{Li}^{\mathrm{b}}$, Jan Youtie ${ }^{\mathrm{b} *}$ and Philip Shapira ${ }^{\mathrm{b}, \mathrm{c}}$
}

July 2016

\footnotetext{
${ }^{a}$ VTT Technical Research Centre of Finland, Espoo, Finland

${ }^{b}$ Georgia Institute of Technology, Atlanta, USA

${ }^{c}$ University of Manchester, Manchester, UK
}

*Corresponding author: jan.youtie@innovate.gatech.edu

Author Accepted Manuscript of paper published in the 


\begin{abstract}
Delineating the emergence of nanotechnologies that offer new functionalities is an important element in an anticipatory approach to the governance of nanotechnology and its potential impacts. This paper examines the transition to next generation active nanotechnologies which incorporate function that respond to the environment or systems concepts that combine devices and structures that are dynamic and which may change their states in use. We develop an approach to identifying these active nanotechnologies and then use bibliometric analysis to examine the extent of research papers and patents involving these concepts. We also examine references to environmental, health and safety concepts in these papers, given that these next generation nanotechnologies are likely to have risk profiles that are different from those of first-generation passive nanomaterials. Our results show a steady growth overall in focus on active nanotechnologies in the research literature and in patents over the study period of 1990 to 2010. We also find an increase in consideration given to environmental, health, and safety topics. While gaps are highlighted in our understanding of research and innovation in active nanotechnologies, the results suggest that there is beginning to be a shift to active nanotechnologies, with the implication that governance processes need to be conscious of this shift and to prepare for it.
\end{abstract}

Keywords: active nanotechnology; systems of nanotechnology; bibliometrics; environmental, health, and safety 


\section{Introduction}

There has been much interest in how nanotechnology research and commercialization will unfold. In recent decades, nanotechnology research funding and scientific publication output has expanded greatly in many countries around the world (Kostoff et al. 2007; Shapira and Wang 2010; Grieneisen and Zhang 2012), and there has also been growth in corporate nanotechnology patenting (Dang et al. 2010; Shapira et al. 2011; Kay et al. 2014). A broad range of nanoscale materials have been engineered and there are numerous products now on the market that are in some way enabled by nanotechnology (Charitidis et al 2014; Vance et al. 2015). Yet, there is also the realization that we are still at the early stages of nanotechnology development and application: more advanced nanotechnology designs, including novel applications that join nanotechnology with bio- and information technologies, have yet to fully emerge out of labs and into the market (Roco et al. 2010; OCED 2014). While there has already been considerable debate about the environmental, health, and safety implications of nanotechnology (see, for example, Royal Society 2004; NRC 2012; Shatkin 2013), it would seem that the entry and responsible innovation of more advanced nanotechnologies that are potentially more disruptive will necessarily require further attention to issues of risk and governance (Murphy et al. 2016).

In this context of both anticipation and uncertainty about emerging nanotechnology applications and their implications, it is important to try to develop evidence-based approaches which can discern what kinds of nanotechnologies are being research in laboratories and the extent to which commercial interest is being shown in their exploitation. This paper contributes to this task of understanding the unfolding of nanotechnology by developing a method to distinguish between different generations of nanotechnology research. In particular, as explained in detail later in this paper, we develop a bibliometric method to distinguish advanced active nanotechnologies that are dynamic and which can change or evolve their states in use and hence offer novel features as well as heighten concerns about risk and governance implications. Although complicated by nuances and ambiguities in defining nanotechnologies and their evolution, the paper puts forward a process and keyword search approach to identifying active nanotechnologies. We apply this search approach to datasets of publications and patents. We then analyze the results to provide a window into the extent, growth, applications, and environmental, health, and safety attention associated with emerging active nanotechnologies. 


\section{Background}

There have been many studies that have contemplated the emergence of nanotechnology and which seek to foretell how the domain will progress and what the implications might be. In the mid-1980s, after the development of scanning tunneling microscopy which enabled atomic-level imaging and manipulation (Baird and Shew 2004), Drexler (1986) envisaged an era of nanotechnology where atoms and molecules could be assembled to create novel mechanisms and structures including molecular machines and self-replicating robots. Debate ensued about the feasibility of molecular assembly and self-replication (Smalley 2001) and its potential environmental and societal risks (see O'Mathúna 2009). While initial debates about the direction of nanotechnology were often at a high and abstract level, subsequently more disaggregated developmental strategies were formulated. In the 1990s and early 2000s, government, academic, and industry groups delivered studies predicting a wide range of applications with novel properties resulting from science and engineering at the nanoscale including in coatings, high-performance materials, electronics, drug delivery, energy efficiency, environmental remediation, and national security (NSTC 1999; OSTL 2002; MANCEF 2004; Royal Society 2004; UT-Battelle 2007). Public and private investment in nanotechnology research and innovation accelerated in the 1990s and in the 2000s (Cientifica 2011; Ouellette 2015). Following the announcement of the US National Nanotechnology in 2000, more than 60 countries established national nanotechnology programs (Shapira and Wang 2010) and, through to the present decade, there has been a massive worldwide growth in nanotechnology publications and patents (Arora et al. 2013; Dang et al. 2010). The applications of nanotechnology to date have mostly resulted in incremental advancements in such areas as semiconductor chips, displays, automotive components, lightweight materials, cleansers, paints and coatings, and catalytic agents (Ouellette 2015; Sargent 2016). Yet, more revolutionary applications continue to be anticipated in medical, energy, environmental, electronic, and other domains (Sargent 2016).

Among efforts to chart future development pathways for nanotechnology is the multi-phase model of successive generations of nanotechnology research and commercialization put forward by MC Roco (2004). In the first phase of this model, there are passive applications of nanotechnology where functionality is fixed, for example in the engineered nanomaterials used in nanocoatings. In a second phase, active applications of nanotechnology emerge. This is followed by further stages of systems of nanosystems and of molecular nanosystems, and an ultimate phase that integrates nanotechnology with information technology, cognitive science, and biotechnology 
(Roco 2004, Renn and Roco 2006). While expected achievement years are indicated in the model, in this paper we are concerned not so much with roadmap scheduling but with the conceptualized technology distinctions that are offered. In the model, the appearance of active nanotechnologies marks a significant shift from an emphasis on individual engineered nanotechnology materials toward conceptualizations of more complex nanotechnology devices and structures, dynamism in use, and the interlinking of non-uniform components. For example, in the catalysis sub-domain, a phased progression from dispensing particles to cages on particles to nanomotors arrayed in a longer chain is envisioned. In the bioscience sub-domain, the envisioned progression is from biocompatible nanostructures to targeted drugs and eventually to nano-electronics embedded in engineered tissue (Scudellari 2012). The work of the Lieber group on nanowire bioactive scaffolds exemplifies the Roco conceptualization of the progression to the systems level in the bioscience sub-domain (Tian et al., 2012). In the electronics sub-domain, the progression might move from insulation to sensors to 3-D and post-CMOS nanoelectronics. Tour (2007) similarly suggests that the use of carbon nanotubes in rubber is an example of the passive phase; nanocars are an example of the active phase; and there is a hybrid phase between the two.

The proposition that nanotechnology research and innovation will undertake an initial transition from passive materials to active devices and systems raises the following duality. On the one hand, the emergence of active nanotechnologies suggests that applications with novel functionalities are more likely to appear, signaling perhaps that at least some of the groundbreaking promises that have long been associated with the narrative about nanotechnology will be realized. On the other hand, the emergence of active nanotechnology applications able to evolve their states raises increased concerns about environmental health and safety risks as well as regulatory and governance procedures (Subramanian et al., 2010). While there has been research on the environmental, health and safety implications of current engineered nanomaterials, the National Research Council has highlighted a lack of research on the environmental, health and safety effects of next generation nanomaterials (NRC 2012).

In 2006, the US National Science Foundation issued a program solicitation for "Active Nanostructures and Nanosystems" of which $\$ 30$ million was awarded to more than 50 projects. However, research activity on active nanotechnologies can be expected to extend much beyond this single program both in the US as well as in other countries. In the science-driven domain of nanotechnology, assessing the extent to which nanotechnology research, in both public and private entities, is shifting towards a focus on active nanotechnologies can offer indications as to 
which pathways are being explored from the view of subsequent commercial applications. In turn, these insights can inform and provide stimuli for deliberation on governance approaches in response to these future pathways for nanotechnology. Guston and Sarewitz (2002) use the term anticipatory governance to describe the capability to responsibly manage such emerging knowledge-based technologies before they become enshrined in widely diffused commercial products.

A core challenge, however, in efforts to anticipate the emergence of active nanotechnologies is that of distinguishing such technologies from those which might be characterized as passive nanotechnologies. Much of the definitional work undertaken to date has focused on developing basic definitions of nanotechnology for research, standards development and governance. The definitions tend to describe vocabularies of nanotechnologies and highlight the importance of a nanotechnology component or dimensional size, typically within the range of 1 to 100 nanometers (BSI 2005, ISO 2008). Functionality in use is less considered. For example, Klaessig's involvement with international standards discussions leads him to conclude that in nanotechnology definitions, cause and effect are disconnected, thus making it difficult for federal agencies to apply oversight (Klaessig et al., 2011; Klaessig 2013). Fatehi and colleagues (2013) likewise agree that there is a lack of agreement on nanotechnology definitions for regulatory and societal purposes. Where regulatory definitions exist, they operate at the level of the nanoparticle.

Alongside definitions of nanotechnology in the regulatory and standards domains, there have also been multiple bibliometric efforts to define nanotechnology. As discussed later in this paper, one replicable bibliometric definition of nanotechnology is that of Porter and colleagues (2008). This definition applies a multistage set of search terms around nanomaterials, instruments, processes, applications and journals and excludes size-based and non-manmade-based references. This definition was subsequently updated to incorporate more materials and exclude additional instruments, the use of which has broadened beyond nanotechnology (Arora et al., 2013). Huang and colleagues found that the various bibliometric definitions have converged in terms of the articles that they extract with their search strategies (Huang et al., 2011). This convergence is due in part to the greater use of nanoprefixed terminology by scientists in their articles' titles, abstracts, and keywords (Arora et al., 2014). At the same time, while these studies develop approaches that broadly capture the scope of nanotechnology, less attention has been paid in bibliometric work to the issue of functionality, and methods to distinguish active nanotechnologies in scientific papers and patents are not well developed. 
The objective of this paper is to develop an explicit and replicable bibliometric definition that can distinguish active next-generation nanotechnologies. Our approach seeks to identify active nanotechnology concepts that respond to the environment (i.e., active nanostructures) as well as systems concepts that combine devices and structures that are dynamic, and appear in a non-uniform manner (i.e., systems of nanotechnology). We are particularly interested to explore which specific categories of active nanotechnology topics are emerging, for instance in convergent areas such as bionanotechnology. We use bibliometric analysis to examine the extent of nanotechnology research papers and patents involving these active concepts. We also examine the take-up of environmental, health and safety aspects in active nanotechnology research papers, in keeping with the NRC's highlighting of the need for attention to the environmental, health and safety implications of next generation engineered nanomaterials. Our methodological approach is detailed in the next part of the paper, followed by a discussion of the analysis and results.

\section{Approach}

We combine a series of methods to identify and enumerate active nanotechnology research. These methods are pursued through the following steps. The initial formation of relevant keywords is based on topics suggested by earlier studies, a key informant interview, and iterative testing. Bibliometric methods are used to collect evidence from scientific publication records. From an ontological perspective, we recognize that a variety of terms of increasing specificity are used in nanotechnology, and that this may influence the frequency of their presence in the data. To ensure a robust approach, further iterations of testing, review and judgment were undertaken to develop the ontology and to select the final combinations of active nanotechnology keywords and topics. Using text mining, these terms are applied to a base dataset of nanotechnology publication records, with further validation, manual term clustering, and exclusion strategies used to derive a subset of active nanotechnology publications for further analysis. (See Figure 1.) This approach builds on the iterative methods of delineating emerging nanotechnologies presented in Porter et al. (2008) and Arora (2013) and is comparable to the evolutionary querying style for nanotechnology tracking put forward by Mogoutov and Kahane (2007).

\section{[Figure 1 about here]}


As a base source of nanotechnology publication records to iteratively test and refine our active nanotechnology search approach, we use the Georgia Tech Global Nanotechnology dataset. This database was developed using the search strategy described in Porter et al. (2008). The search strategy is based on a multi-stage Boolean approach involving a two-step inclusion and exclusion process. The key terms used for inclusion and exclusion were formulated in conjunction with nanotechnology experts. The search strategy and its results have been used in numerous studies of nanotechnology research and innovation, including academic and policy studies, and has performed robustly in comparison with other nanotechnology search approaches (Huang et al., 2011). The search strategy was updated by Arora et al. (2013), and we use a dataset produced by this refreshed approach in the current study. Our base nanotechnology dataset comprises 757,868 nanotechnology publication records from the Web of Science for 1990-2010. Of these records, 94\% are articles or proceedings papers. This dataset is global in scope and it encompasses a critical two-decade period which incorporates the early emergence of nanotechnology research in the early 1990 s and its subsequent growth in scale, complexity, and attention to applications in the 2000s (Youtie et al, 2008; Shapira \& Wang, 2010). While not incorporating more recent years, this two-decade period provides a basis of large-scale data over a sufficiently lengthy period of time to bibliometrically test whether it is possible to delineate the emergence of research on active nanotechnologies. The Web of Science is commonly used in this kind of bibliometric analysis. We acknowledge that in addition to its strengths, including broad worldwide coverage of journals across all fields of science and technology and structured record format, there are also limitations, such as the level of inclusion of non-English language science journals (see comparison of publication databases in Falagas et al., 2008).

Our methodology is informed by a previous study (Subramanian et al., 2010) that investigated the extent of any shift in nanotechnology research from passive to active nanostructures. (The third and fourth authors of the present study were also involved in the Subramanian work.) In the Subramanian study, a two-stage process was used. First, a term set associated with active nanotechnologies, including terms related to actuation, motor, rotor, sensing and nanoelectromechanical systems, was applied to a bibliometric database of nanotechnology publications. It was found that scientific publications in active nanotechnology increased markedly after 2006. Second, a bibliographic review of the abstracts of a sample of the identified active nanostructure publications was undertaken. This personal review identified five not-mutually exclusive sub-segments in active nanostructure research: (1) remote actuated, where the active element is remotely activated or sensed; (2) environmentally responsive, where 
the active element is sensitive to stimuli such as $\mathrm{pH}$ or certain chemicals; (3) miniaturized active, where active components of larger devices are scaled down to the nanoscale; (4) hybrid active, involving uncommon combinations such as organic-inorganic; and (5) transforming active, involving nanotechnology that changes irreversibly during use.

Our current analysis not only updates this earlier search but also advances the methodology by significantly extending the approach to delineating and testing search terms. The earlier Subramanian work depended significantly on a personal reading of abstracts. While attention was given to identifying active nanostructure search terms, the key word set was limited and this initial work did not incorporate the insights from the abstract review to enhance the active nanostructure search terms. In contrast, the analysis detailed in the present paper is based primarily on the iterative identification, testing and application of active nanotechnology search terms to abstracts, titles, and keywords of nanotechnology publications. This approach enables improved recall - the ability of a bibliometric search query to encapsulate a high proportion of the relevant publication records - with a reasonable level of precision to reduce the entry of irrelevant records (Buckland and Gey, 1994). It is also an approach that facilitates reproducibility and updating, as the key words that we apply can be reprised and refined in subsequent studies. At the same time, we acknowledge that there is inevitably a measure of subjectivity in determining what should be categorized as active nanotechnology. Moreover, some of the concepts associated with active nanotechnology may not yet have evolved and diffused into distinct keywords that can be easily identified in extant scientific publications. In recognition of these limitations, the approach allows for judgement and a degree of latitude in accepting the papers returned by certain keywords in the search query.

In addition to our re-appraisal of Subramanian, a further preliminary step in the process to derive an improved active nanotechnology bibliometric query was to review the formative paper by Renn and Roco (2006). This discusses early passive nanostructures and anticipated later generations of active nanostructures, systems of nanosystems, and molecular nanosystems. A series of categories and examples of potential products are offered for each of the four nanotechnology generations presented in this paper. The authors acknowledge that there is overlap between the generations. Nonetheless, a reading of this paper suggests a series of terms that can be used to distinguish between passive and active phases and types of nanotechnology. In subsequent interviews with Roco, 
we further probed how the different phases of nanotechnology could be delineated. ${ }^{1}$ This led to an initial and tentative list of potential keywords to represent active nanotechnology. We then tested these keywords against the Georgia Tech Global Nanotechnology dataset and examined the returns received. This was done both through reading selected abstracts of publications and co-word visualization of active nanotechnology identifiers.

The tentative list of potential keywords included simple terms such as active nano or active nanotechnology as well as modifiers such as adaptive or self-healing that promised to convey characteristics of movement, interrelatedness, and dynamism associated with active nanotechnology. In our testing, we found that keywords such as motor and rotor applied to our base nanotechnology dataset produced abstracts which corresponded with active nanotechnology concepts. Terms such as systems, juncture, dynamic, and interface were found to be too general. The phrase active nanotechnology was not widely used. Other terms were too specific, for example, wire electrode arrays, or did not yield many publications, for example impeller. Still other terms - antenna, wireless, or adaptivegenerated papers that largely were not in the active nanotechnology domain.

We continued our search for appropriate keywords by focusing on motor and rotor, and used string matching and co-word visualization (through network maps of terms occurring together with motor or rotor) to highlight additional terms for consideration (Figure 2). These visualizations were undertaken using SPSS text analytics software. The similarity metric, as presented legend in the "motor" and "rotor" co-word visualization, measures the strength of co-occurrence links: higher values (indicated by thicker lines) indicate concept pairs appearing more frequently together than apart. (For further details about this software and the similarity metric formula, see:

ftp://public.dhe.ibm.com/software/analytics/spss/documentation/modeler/15.0/en/Users_Guide_For_Text_Analytics. pdf, p. 143.) The co-word visualization associated with rotor brought forth molecular terms for consideration while that for motor pointed to terms such as cargo and kinesin and other proteins. We further investigated co-words associated with these terms which highlighted terms such as biosensor, molecular switch, tweezer, shuttle, nanovalve, logic gate, nanoelectromechanical system or NEMS, and nanorobot. These terms were then formed into an ontology which classified terms according to their activities (e.g., biosensing, kinesis, molecular assembly, responsive), functional parts (actuator, nanocar (not nanocarbon), switch (molecular), motor (molecular), rotor,

\footnotetext{
${ }^{1}$ Interviews were conducted with Mihail C. Roco by telephone on September 7, 2012, and in-person on February 18, 2013 at the National Science Foundation, Arlington, VA.
} 
tweezers/clips (molecular), shuttle, nanovalve, logic gate, transistor, nanoelectromechanical system or NEMS, and systems (including robot but not robotics). The ontology also included some proper nouns which identify particular proteins or molecular architectures with active nanotechnology characteristics such as rotaxenes, catenanes, kinesin, and dynein.

\section{[Figure 2 about here]}

After further review, the resulting keyword set was organized into four main subgroups (Table 1). The first subgroup comprised terms for biosensing and related activities. The second subgroup comprised functional parts including aspects of molecular electronics such as molecular switch, molecular tweezers, motor, and rotor. This group also included the keyword, transistor, which is commonly associated with single molecular electronics or engineered nanoparticle electronics (such as graphene transistors) as well as standard characterizations of engineered phenomenon at the nanoscale (as in FET). We acknowledge the difficultly of distinguishing between the two simply through the use of keywords but elected to retain transistor because of the number of papers associated with the term that are in the active nanotechnology domain. The third subgroup of proper nouns contained proteins with characteristics associated with active nanotechnologies. The fourth subgroup encompassed papers associated with systems of nanotechnology through a focus on nanorobots.

\section{[Table 1 about here]}

Once the active nanotechnology keyword set was finalized, we undertook an analysis of the publication records produced when the terms were applied to the base dataset of nanotechnology records. Data cleaning, including de-duplication of records and addressing inconsistencies in author, organization, journal title, and other fields, was accomplished with VantagePoint software (www.thevantagepoint.com). VantagePoint was also used for basic analysis and descriptive tabulation. Network relationships were characterized using Pajek, a freely-available software tool for the analysis and visualization of complex networks (http://mrvar.fdv.uni-lj.si/pajek/). We used R, a free statistical software package, to perform topic modeling (https://www.r-project.org/).

\section{Analyses and Results}

Once our active nanotechnology search approach was completed, several analyses were undertaken. First, we began with an examination of the number of active nanotechnology publications over time and their growth rate. 
If there is indeed a shift in focus underway from passive to active nanotechnology research, we would expect to see relatively higher growth in active nanotechnology publications. Second, we investigated active nanotechnology publications by identified terms and other characteristics including their association with certain nanomaterials. Here, we sought to discern what kinds of active nanotechnologies were attracting most research attention, whether there were relationships and clusters among various active nanotechnology research topics, and what engineered materials were most likely to be associated with active nanotechnology research. Third, we analyzed whether and how active nanotechnology publications also included reference to environmental, health, and safety concepts and potential implications. This is relevant given the different and potentially greater risk profiles associated with active nanotechnology devices and systems. Finally, given the expectation that advanced active nanotechnologies will enter commerce over the next decade (NRC 2012, p. 31), we also explored interest in exploitation by investigating the presence and applications of active nanotechnologies in patent databases.

Growth of active nanotechnology publications. Using the active nanotechnology keyword set, and following data cleaning, we identified more than 46,000 active nanotechnology publications issued between 19902010 within our global nanotechnology dataset, or $6.1 \%$ of all nanotechnology publications. In the earlier Subramanian work, about 29,000 active nanotechnology papers were identified between 1995 and 2008, equivalent to about $4.1 \%$ of the reference nanotechnology database for that period, with a takeoff in 2007-2008. Different methods are used in the two studies (see earlier discussion), with the current study using an elaborated approach that has advantages in identifying active nanotechnology papers. Using this elaborated approach, it does seem that active nanotechnology publications have achieved a relatively strong growth rate, continuing through to the most recent years of the study. We observe steady growth rather than any sharp take-off point (suggesting that there were influences other than the 2006 NSF active nanotechnology program in stimulating growth in this segment). As a percentage of all nanotechnology publications, the active nanotechnology share rose from 3\% in 1991 to $8 \%$ in 2010 (Figure 3). The average annual growth rate from 1991 to 2010 for active nanotechnology is $130 \%$ versus $55 \%$ for all nanotechnology publications. In the more recent 2006-2010 period, active nanotechnology publications continued to maintain a slightly faster recent annualized growth rate: $19 \%$ from $2006-2010$ versus $11 \%$ for all nanotechnology publications. Within the broader domain of nanotechnology, we do find that there is a shift in research towards active nanotechnology topics. However, we do not observe any noticeable breaks or take-offs in the number or share of active nanotechnology publications, rather the distribution appears to move upwards in a continuous fashion. 
[Figure 3 about here]

Characteristics of active nanotechnology research. One way to examine this topic is by investigating the breakdown of publications by the different search terms we used to define the domain. Terms associated with the largest number of active nanotechnology publications are: transistor (nearly $40 \%$ of papers), biosensing/biosensor (33\% of papers), and motor (12\% of papers). Actuator, valve and kinesin comprise from $3 \%$ to $5 \%$ of the publications. Two percent of the papers involve any of the following: rotaxane, nanocar, NEMS, rotor, or logic gate, and another one percent involve molecular switch, dynein, or catenane. Least common, comprising less than one percent of the papers, are nanorobot and molecular tweezers/clips.

To probe the underlying configuration of active nanotechnology publications, we examined the connections between these search terms. A network diagram was developed to examine the underlying relationships between these keywords or keyword co-occurrence as a proxy for sub-groups in the active nanotechnology domain (Figure 4). Although the position of the keywords/nodes has no meaning, the thickness of the links in the network diagram represents the percentage of shared counts of the node associated with the smaller numbers of publications. The network diagram indicates that only a few terms have very strong relationships. We interpret these relationships as reflecting sub-groups of active nanotechnology:

- The first sub-group is reflected in the very strong linkage between kinesin, dynein, and motors. These terms constitute the basic elements of molecular motors.

- The second sub-group includes catenane and rotaxane. These are the foundations for molecular structures such as switches and carriers.

- Constituent parts of nanoelectronics such as transistors and logic gates characterize a third sub-group.

- A fourth element - biosensors - has a number of weak links. This pattern suggests the involvement of a range of active nanotechnologies in drug delivery and other aspects of nanobiotechnology.

\section{[Figure 4 about here]}

These sub-groups also reference other more complex structures and devices. For example, DNA origamithe self-assembly of DNA components into folds with distinctive interactions with light (Griggs, 2012) - is featured in 11 articles in the active-and-beyond papers. This total does not represent all DNA origami papers, but rather those 
works that fall into the active nanotechnology publication domain. More than one quarter of these papers involve motors, and another similar share involves either transistors or actuators.

A further aspect of the underlying character concerns the relationship of these terms to engineered nanomaterials. Nearly one-third of the active nanotechnology publications make reference in their titles, abstracts, or keywords to engineered nanomaterials. We define engineered nanomaterials using the list of these materials in Table 3-2 of the National Research Council report (NRC 2012). The most commonly mentioned engineered nanomaterial is gold nanoparticles (in 6,952 publications in our active nanotechnology dataset), followed by carbon nanotubes $(4,178$ publications). Some active nanotechnology devices and structures and devices are more likely to incorporate engineered materials than others. Nearly half of all biosensor articles also mention an engineered nanomaterial; however, only $6 \%$ of motor articles mention an engineered nanomaterial. Active nanotechnology publications that reference transistors and actuators most commonly also reference carbon nanotubes, fullerene, graphene, and quantum dots. Gold, silver, and platinum nanoparticles as well as carbon nanotubes are commonly referenced in biosensor-related active nanotechnology publications (Table 2).

\section{[Table 2 about here]}

We probed further into the underlying characteristics of active nanotechnology by using topic modeling. Topic modeling is based on Bayesian probabilistic models that use observed words in documents to uncover the unobserved structure that underlies a set of documents (in this case, journal article meta data) indicated in the probability distribution of words to $\mathrm{K}$ topics and the probability distribution of $\mathrm{K}$ topics to $\mathrm{M}$ documents in a corpus (Blei, 2012). In essence, the approach uses these distributions to establish which words belong together to form topics. An advantage of topic modeling is that it allows the identification of relevant documents drawing on synonyms as well as keywords. Topic modeling via Latent Dirichlet Allocation (LDA) was applied to title, keyword, and abstract phrases in the active nanotechnology publication dataset. We used the R statistical computing language to perform topic modeling. Twelve topics resulted and we associated each publication with one or more topics. We observe that the topical emphases of active nanotechnology have changed over time (Figure 5). In the early 1990s, the optic, layer, nanowire topic was prevalent. The later 1990s saw the rise of the electrode, biosensor, enzyme topic, highlighting the integration at the nano scale of biologically- and chemically-based sensors with electronic signaling devices. The quantum, magnet, electron, field topic rose to prominence in the early 2000 s. By the end of 
the 2000s, there were two groups of topics with different trajectories. Topics related to nanoelectronics (e.g., film, layer, deposit) had a moderate growth pattern while topics related to biosensors (e.g., electrode, biosensor, enzyme and detect, sensor, DNA, signal, hybrid) had a more rapid upward trajectory. These results suggest that bionanotechnology has become especially important in the active nanotechnology domain.

[Figure 5 about here]

Consideration of environmental, health and safety. The devices and systems associated with active nanotechnology are said to be associated with different (and potentially more significant) risk profiles than passive nanotechnology materials (IRGC 2007). While it is outside the scope of this paper to assess the specific nature of any risks, we do investigate the extent to which active nanotechnology research explicitly considers aspects associated with environmental, health, and safety (EHS). We do this by examining references to EHS in the titles, abstracts and keywords of active nanotechnology publications. The EHS definition we use is described in Youtie et al. (2011). This bibliometric definition of EHS is comprised of three elements. The first relates to terms linked to the "nano" prefix such as nanotoxicity, nanoecotoxicity, and nanosafety. The second is comprised of broader EHS terms that are conditioned with other terms such as hazard, risk, dose, and exposure. The third are terms to be excluded because of being associated with too much noise such as safety glass or potentially being beneficial or neutral (rather than detrimental) such as antimicrobial and nontoxic.

Nearly $4 \%$ of active nanotechnology publications refer to EHS terms, and this percentage grew from $2 \%$ in the 1990 s to nearly $7 \%$ by 2010 . While these percentages appear low, they are higher than in the larger Georgia Tech reference nanotechnology publication dataset where, based on a comparable EHS analysis, fewer than 1\% of the papers refer to EHS terms. Active nanobiotechnology publications were more likely to also have a reference to EHS terms. Almost one-third of active nanotechnology publications with the term nanocarrier also referenced an EHS term. Next most prevalent were nanorobot and biosensor, with respectively $7 \%$ and $6 \%$ of these publications also referencing an EHS term. Since nanocarriers are typically linked to applications in the human body, it is makes sense that EHS considerations are more frequently also expressed in these research publications. Overall, there appears to be modestly increased recognition of potential EHS implications in published active nanotechnology research compared with other nanotechnology work. That the great majority of published active nanotechnology research does not make reference to EHS aspects could be interpreted in different ways. Much of this research is likely to be fundamental science, where applications (if any) are yet to be determined. On the other hand, as research 
becomes more developed and applied, it could be argued that researchers should give greater attention to potential EHS implications.

Interest in exploitation through patenting. To test the extent to which active nanotechnologies are considered as potentially exploitable, we also examined the presence of active nanotechnology concepts in patents. Global nanotechnology patent data was extracted by applying the Georgia Tech nanotechnology search strategy (as detailed in Arora et al., 2013) to PATSTAT - a worldwide database of patent records from patent offices in about 90 countries that is maintained by the Europe Patent Office (EPO, 2016). The keywords used to identify active nanotechnology research papers were then applied to patent titles and abstracts in the global nanotechnology patent dataset. Upon manual review of the resulting titles and abstracts, we found that some active nanotechnology keywords did not translate well in patent searches. In response, we developed several additional exclusion terms to cull out nanotechnology patents that were not in the active nanotechnology domain but which referenced motor vehicles, automobiles, nanocarbon (as a material), and semiconductors. The end result was an active nanotechnology patent dataset of 7,925 patent records (applications and grants) from 1990 to 2012. This figure represented about 4\% of the 194,077 patent records in the Georgia Tech dataset for this time period. When compared with active nanotechnology publications, we observe approximately six active nanotechnology publications papers for every one patent record.

Twenty-eight percent of active nanotechnology patent records have non-patent references to scholarly literature. This same percentage for all nanotechnology patents in the Georgia Tech global nanotechnology patent database is 23 percent. The active nanotechnology patent subset is more likely to have non-patent references to scholarly literature than that for all nanotechnology patents. Our analysis of active nanotechnology patent records indicates that more than $40 \%$ relate to transistors. Twenty percent involve biosensors. The next most common are active nanotechnology patent records that reference motor $(14 \%)$, valve $(11 \%)$, actuator (9\%), rotor (5\%), and nanocar/nanocarrier (2\%). Interest in exploiting active nanotechnology thus encompasses a mix of nanoelectronics, nanobiotechnology, and engineered materials.

Nearly $62 \%$ of these active nanotechnology patents are assigned to companies. Holding a patent does not necessarily mean that this intellectual property will be incorporated into a specific product. However, patent applications and grants broadly suggest there is current or planned corporate interest in active nanotechnologies. The 
top assignees for transistor patents are: NEC, Samsung, and IBM (each with 4\% of these patents). The top assignees for biosensor patents are: Fuji (5\% of these patents), Matsushita (3\%), Philips (2\%), and Kimberly Clark (2\%). While larger firms account for most of the assignees with 10 or more patents in this domain, some smaller nanofocused firms also are present, for example, Nanosphere, Inc. (with 14 patents in the domain).

\section{Conclusions}

In this paper, we detail the iterative development and testing of a keyword-based bibliometric approach to identify active nanotechnologies. We then applied these keywords to measure and assess research and patenting in active nanotechnology domains. We found, over our study period through to 2010 , increasingly higher growth in the rate of active nanotechnology publications and a relative increase in the share of active nanotechnology research among all nanotechnology publications. This suggests that there is beginning to be a shift in focus from passive to active nanotechnology research, although as yet the absolute share of active nanotechnology in all nanotechnology research is still relatively modest. In examining the characteristics of active nanotechnology research, we observed steady yet upward growth in publications involving multiple research dimensions including biosensors, molecular electronics in transistors and logic gates, protein-based molecular motors, and molecular structures for switches and carriers. Active nanotechnology researchers were more likely than other nanotechnology researchers to incorporate EHS concepts, particularly in nanocarrier work for nano-enabled drug delivery. Still, overall, the proportion of active nanotechnology papers referencing EHS implications remained relatively low. Active nanotechnology concepts were shown to be present in a small but not insignificant set of patent records, more than three-fifths of which were assigned to corporations, suggesting that active nanotechnology concepts, materials, and systems are attracting interest among private entities engaged in commercializing new technologies.

This exploratory effort to provide a quantitative and systematic basis for understanding the trajectory of active nanotechnology development has provided multiple insights. Nonetheless, gaps in our understanding and knowledge remain. In the context of ongoing definitional debates about size, distinctive properties, and coherence of the field of nanotechnology writ large (see for example, Schummer 2014), attention also needs to be paid to distinguishing specific phases and directions in the field, including, for example, the emergence of active nanotechnologies. It is important to probe the mechanisms underlying the generations of nanotechnology development and how these result in active, kinetic, dynamic, and/or integrative properties. Additionally, ongoing 
efforts are needed to understand how the lexicon used to identify active nanotechnologies continues to evolve as scientific progress advances the state of the art in materials, devices and systems. In this paper, our contribution has been to advance bibliometric approaches that can applied to delineate phases and emerging research directions of nanotechnology development.

In reflecting on these issues, we do not find that it is enlightening to focus too exclusively on engineered nanomaterials. While the NRC (2012) report places an emphasis on certain engineered nanomaterials, we observe that active nanotechnologies have an orientation towards interrelationships and to embedding engineered materials into systems involving other emerging technologies. Indeed, our analysis shows that several active nanotechnology concepts can co-occur in a given research publication. Proteins co-occur with motors, mechanically interlocked molecular architectures are investigated together under the search for switches and carriers, transistors and logic gates are linked as potential nanoelectronic constituent parts, and biosensors are incorporated into multiple research domains. Treating engineered nanomaterials as separate elements can lead to a misunderstanding in how they will be used in integrated device and systems. Moreover, as hinted by our analysis, there are many ways in which integrated relationships will be embedded into active nanotechnology devices and systems that enter into markets. For example, Zhou and colleagues (2014) show that nano-enabled delivery platforms are grafted onto current pharmaceuticals rather than seeing co-development or multi-functional approaches. More in-depth research into how seemingly active nanotechnologies are, or will be, used and integrated in the commercial sector is surely needed. In addition to improving our business understanding of potential applications, it is responsible to address further attention to the environmental, health and safety implications of active nanotechnology applications. Our results indicate that active- nanotechnologies are steadily emerging both in scientific and commercial domains. The level of activity that we uncovered, and its steady growth, suggests that more extensive consideration should be given to the emergence of active nanotechnologies in governance deliberations and in associated policies. 


\section{Acknowledgements}

We thank David Guston, Kathy Eggleson, and Fred Klaessig for their helpful suggestions on an earlier version of this paper, which was presented at the Workshop on the Anticipatory Governance of Complex Engineered Nanomaterials. This study was undertaken with support from the US National Science Foundation under and Award \# 0937591 (Center for Nanotechnology in Society at Arizona State University), and Award \# 1235693

(Collaborative Research: Workshop on the Anticipatory Governance of Complex Engineered Nanomaterials). Any opinions, findings, and conclusions are those of the authors and do not necessarily reflect the views of the sponsors. 


\section{References}

Arora SK, Porter AL, Youtie J, Shapira P (2013) Capturing new developments in an emerging technology: an updated search strategy for identifying nanotechnology research outputs. Scientometrics, 95(1): 351-370.

Arora SK, Youtie J, Carley S, Porter AL, Shapira P (2014) Measuring the development of a common scientific lexicon in nanotechnology. Journal of Nanoparticle Research 16(1): 1-11.

Baird D, Shew A (2004) Probing the History of Scanning Tunneling Microscopy, in Baird D, Nordmann A, Schummer J, eds. Discovering the Nanoscale. Amsterdam: IOS Press, 145-156.

Blei DM (2012) Probabilistic topic models. Communications of the ACM 55(4): 77. doi:10.1145/2133806.2133826

BSI (2005) Vocabulary - Nanoparticles. PAS 71:2005. London: British Standards Institute.

Buckland M, Gey F (1994) The relationship between recall and precision. Journal of the American Society for Information Science 45(1): 12-19.

Charitidis CA, Georgiou P, Koklioti MA, Trompeta A-F, Markakis V (2014) Manufacturing nanomaterials: from research to industry. Manufacturing Review 1(11):1-19. doi: 10.1051/mfreview/2014009

Cientifica (2011) Global funding of nanotechnologies and its impact. London. July.

Dang Y, Zhang Y, Fan L, Chen H, Roco MC (2010) Trends in worldwide nanotechnology patent applications: 1991 to 2008. Journal of Nanoparticle Research 12(3): 687-706. doi: 10.1007/s11051-009-9831-7.

Drexler KE (1986) Engines of Creation: The Coming Era of Nanotechnology. Anchor Books, New York.

EPO (2016) PATSTAT Online v2.2.8 User Manual. European Patent Office. http://documents.epo.org/projects/babylon/eponet.nsf/0/5AE173034539C62DC1257990004F162F/\$File/pa tstat_online_user_manual_v2.11_en.pdf

Falagas ME, Pitsouni EI, Malietzis GA, Pappas G (2008) Comparison of PubMed, Scopus, Web of Science, and Google Scholar: trengths and weaknesses. FASEB Journal 22:338-342

Fatehi L, Kuzma J, Najmaie P (2013) A Delphi Approach to Finding a CrossDisciplinary Definition of "Nano" for Research, Society, and Regulation. Paper presented at the fifth annual meeting of the Society for the study of Nanoscience and Emerging Technologies, Boston, Massachusetts USA October 27-29, 2013.

Grieneisen ML, Zhang MD (2012) The ongoing proliferation of nano journals. Nature Nanotechnology 7(5): 273 274. doi: $10.1038 /$ nnano.2012.53.

Griggs MB (2012) How DNA Origami Creates Supermaterials. Popular Mechanics. March 16. 
Guston, DH, Sarewitz D (2002) Real-time technology assessment. Technology in Society 24(1): 93-109.

Huang C, Notten A, Rasters N (2011) Nanoscience and technology publications and patents: a review of social science studies and search strategies. Journal of Technology Transfer 36(2):145-172.

IRGC (2007) Nanotechnology risk governance: recommendations for a global, coordinated approach to the governance of potential risks. Policy Brief. Geneva: International Risk Governance Council.

ISO (2008) Nanotechnologies - Terminology and definitions for nano-objects - Nanoparticle, nanofibre and nanoplate. ISO/TS 27687:2008(E). Geneva: International Organization for Standardization.

Kay L, Youtie J, Shapira P (2014) Signs of things to come? What patent submissions by small and medium-sized enterprises say, about corporate strategies in emerging technologies. Technological Forecasting and Social Change 85:17-25. doi:10.1016/j.techfore.2013.09.006.

Klaessig, F. (2013) Setting Useful Nanotechnology Definitions Examined from the Standpoint of Central and Peripheral Claim Language Used in Intellectual Property Law. In Konrad, Coenen, Dijkstra, Milburn, and van Lente, Shaping Emerging Technologies: Governance, Innovation, Discourse. IOS Press, Berlin.

Klaessig F, Marrapese M, Abe S (2011) Current perspectives in nanotechnology terminology and nomenclature. In: Murashov V, Howard J, eds., Nanotechnology Standards. New York: Springer Science+Business Media. $21-52$.

Kostoff RN, Koytcheff RG, Lau, CGY (2007) Global nanotechnology research literature overview. Technological Forecasting and Social Change 74(9): 1733-1747. doi:10.1016/j.techfore.2007.04.004

MANCEF (2004) International Micro - Nano Roadmap. Elders J, Giasolli R, Walsh S eds. Micro and Nano Technology Commercialization Education Foundation, Albuquerque, NM.

Mogoutov A, Kahane B (2007) Data search strategy for science and technology emergence: A scalable and evolutionary query for nanotechnology tracking. Research Policy, 36:893-903.

Murphy F, McAlea EM, Mullins M eds. (2016) Managing Risk in Nanotechnology: Topics in Governance, Assurance, and Transfer. Springer International Publishing.

NRC (2012) A Research Strategy for Environmental, Health, and Safety Aspects of Engineered Nanomaterials. National Academies Press. Washington DC. 
NSTC (1999) Nanostructure Science and Technology: A Worldwide Study. Interagency Working Group on Nanoscience, Engineering and Technology and the National Science and Technology Council, Executive Office of the President, Washington, DC.

OECD (2014) Nanotechnology in the context of technological convergence. Directorate for Science, Technology and Innovation, Working Party on Nanotechnology, DSTI/STP/NANO(2013)10/FINAL. Paris: Organisation for Economic Cooperation and Development. http://www.oecd.org/officialdocuments/publicdisplaydocumentpdf/?cote=dsti/stp/nano(2013)10/final\&docl anguage $=\mathrm{en}$

O'Mathúna DP (2009) Nanoethics: big ethical issues with small technology. Continuum, London.

OSTL (2002) New Dimensions for Manufacturing: A UK Strategy for Nanotechnology. UK Advisory Group on Nanotechnology Applications. UK Office of Science and Technology, London.

Ouellette LL (2015) Nanotechnology and innovation policy. Harvard Journal of Law \& Technology 29(1):34-75.

Porter AL, Youtie J, Shapira P, Schoeneck, D (2008) Refining search terms for nanotechnology. Journal of Nanoparticle Research 10(5):715-728.

Renn O, Roco M (2006) Nanotechnology and the need for risk governance. Journal of Nanoparticle Research 8:153191.

Roco MC (2004) Nanoscale Science and Engineering: Unifying and Transforming Tools. AIChE Journal 50(5): 890-897.

Roco MC, Mirkin CA, Hersam MC (2010) Nanotechnology Research Directions for Societal Needs in 2020: Retrospective and Outlook. WTEC. http://www.nano.gov/sites/default/files/pub_resource/wtec_nano2_report.pdf

Royal Society (2004) Nanoscience and nanotechnologies: opportunities and uncertainties. The Royal Society and The Royal Academy of Engineering, London.

Sargent JF (2016) Nanotechnology: A Policy Primer. Congressional Research Service. RL 34511. Washington, DC. Schummer J (2014). On the novelty of nanotechnology: A philosophical essay. In: In Pursuit of Nanoethics, Springer Netherlands, 15-29.

Scudellari M (2012) Cyborg Tissue Monitors Cells. Technology Review, Monday August 27.

Shapira P, Wang J (2010). Follow the money. Nature 468(7324):627-8. doi:10.1038/468627a 
Shapira P, Youtie J, Kay L (2011) National Innovation Systems and the Globalization of Nanotechnology Innovation. Journal of Technology Transfer 36(6):587-604.

Shatkin JA (2013) ed. Nanotechnology: Health and Environmental Risks. Second Edition. CRC Press, Boca Raton, FL.

Smalley RE (2001) Of Chemistry, Love and Nanobots. Scientific American 285(3):76-7. doi:10.1038/scientificamerican0901-76. PMID 11524973.

Subramanian V, Youtie J, Porter AL, Shapira P (2010). Is there a shift to "active nanostructures"? Journal of Nanoparticle Research 12(1):1-10.

Tian B, Liu J, Jin L, Tsui J, Qing Q, Suo Z, Langer R, Kohane D, Lieber C (2012) Macroporous nanowire nanoelectronic scaffolds for synthetic tissues. Nature Materials. DOI: 10.1038/NMAT3404.

Tour JM (2007) Nanotechnology: The Passive, Active and Hybrid Sides - Gauging the Investment Landscape from the Technology Perspective. Nanotechnology Law and Business 2007 4:361-373.

UT-Battelle (2007) Productive Nanosystems: A Technology Roadmap. Battelle Memorial Institute and the Foresight Nanotech Institute.

Vance ME, Kuiken T, Vejerano EP, McGinnis SP, Hochella MF Jr, Rejeski D, Hull MS (2015) Nanotechnology in the real world: Redeveloping the nanomaterial consumer products inventory. Beilstein Journal of Nanotechnology 6:1769-1780. http://dx.doi.org/10.3762/bjnano.6.181

Youtie J, Porter A, Shapira P, Tang L, Benn T (2011) The use of environmental, health and safety research in nanotechnology research. Journal of Nanoscience and Nanotechnology 11(1), 158-166.

Youtie J, Shapira P, Porter AL (2008) Nanotechnology publications and citations by leading countries and blocs. Journal of Nanoparticle Research 10:981-986.

Zhou X, Porter AL, Robinson DK, Shim MS, Guo Y (2014) Nano-enabled drug delivery: A research profile. Nanomedicine: Nanotechnology, Biology and Medicine 10(5):889-896. 


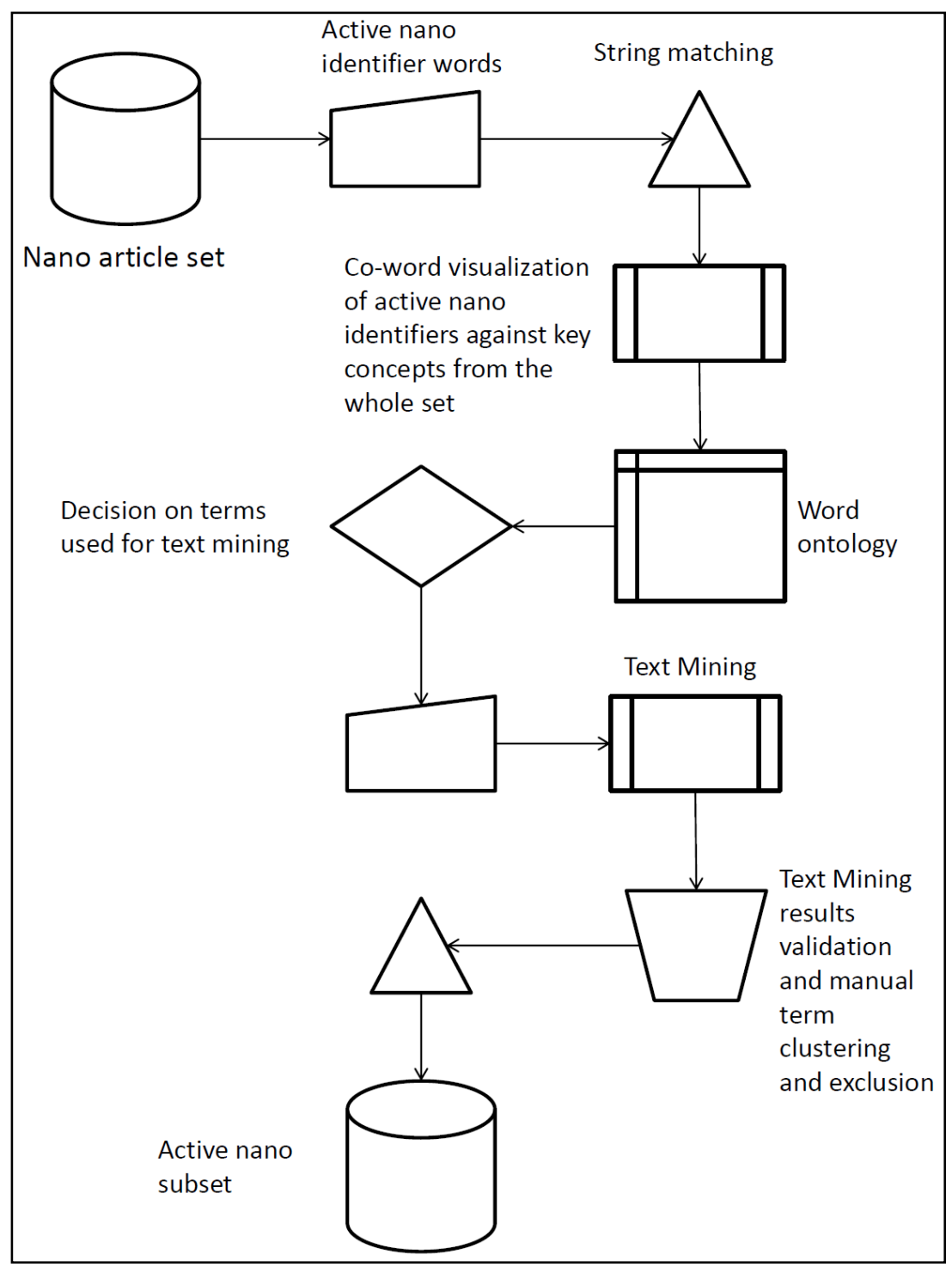

Fig. 1 Bibliometric process to derive active nano article subset 


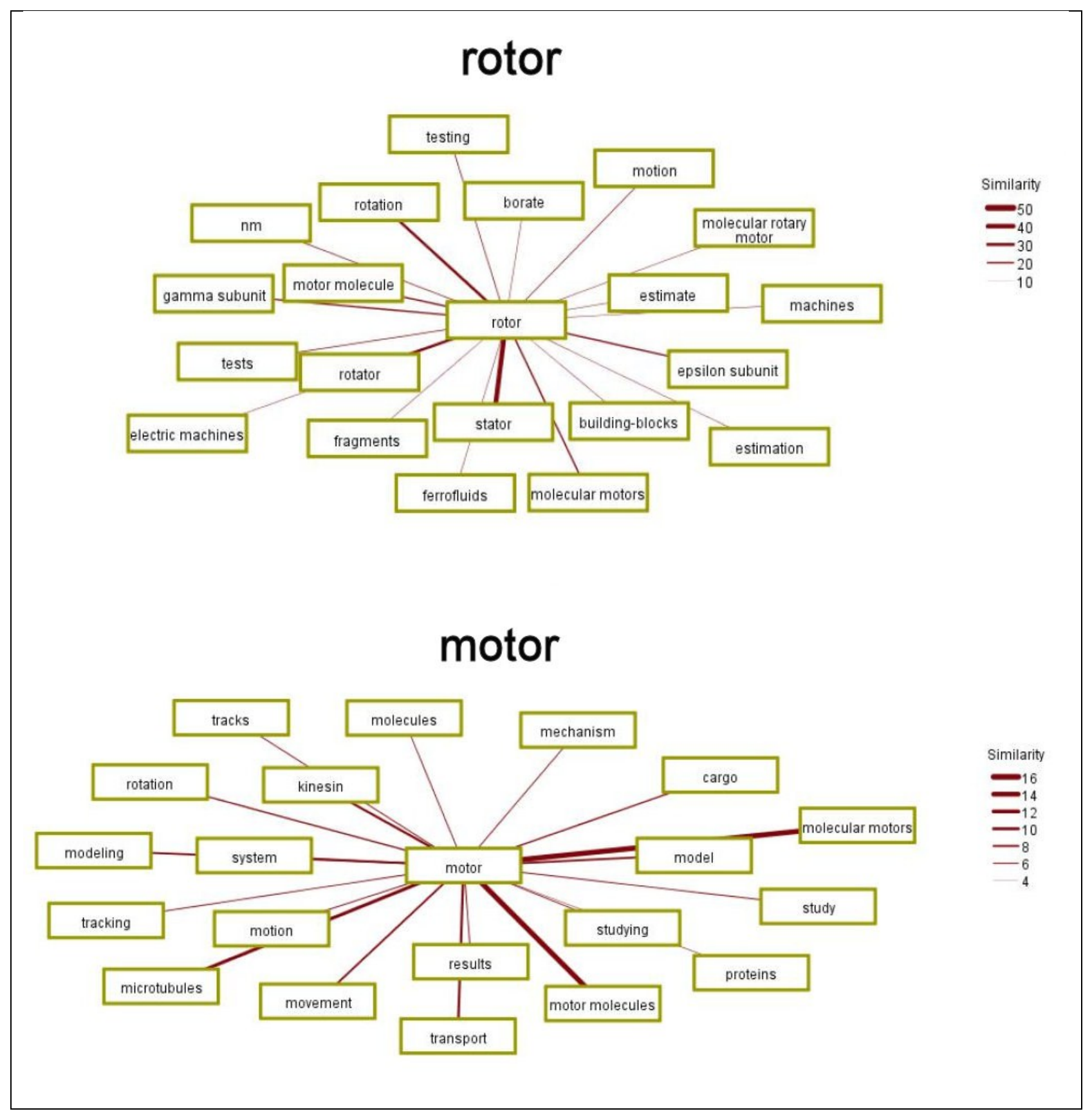

Fig. 2 Co-word visualizations: motor and rotor

Note: Visualizations undertaken using SPSS text analytics software. The similarity metric measures the strength of co-occurrence links. See text for further details. 


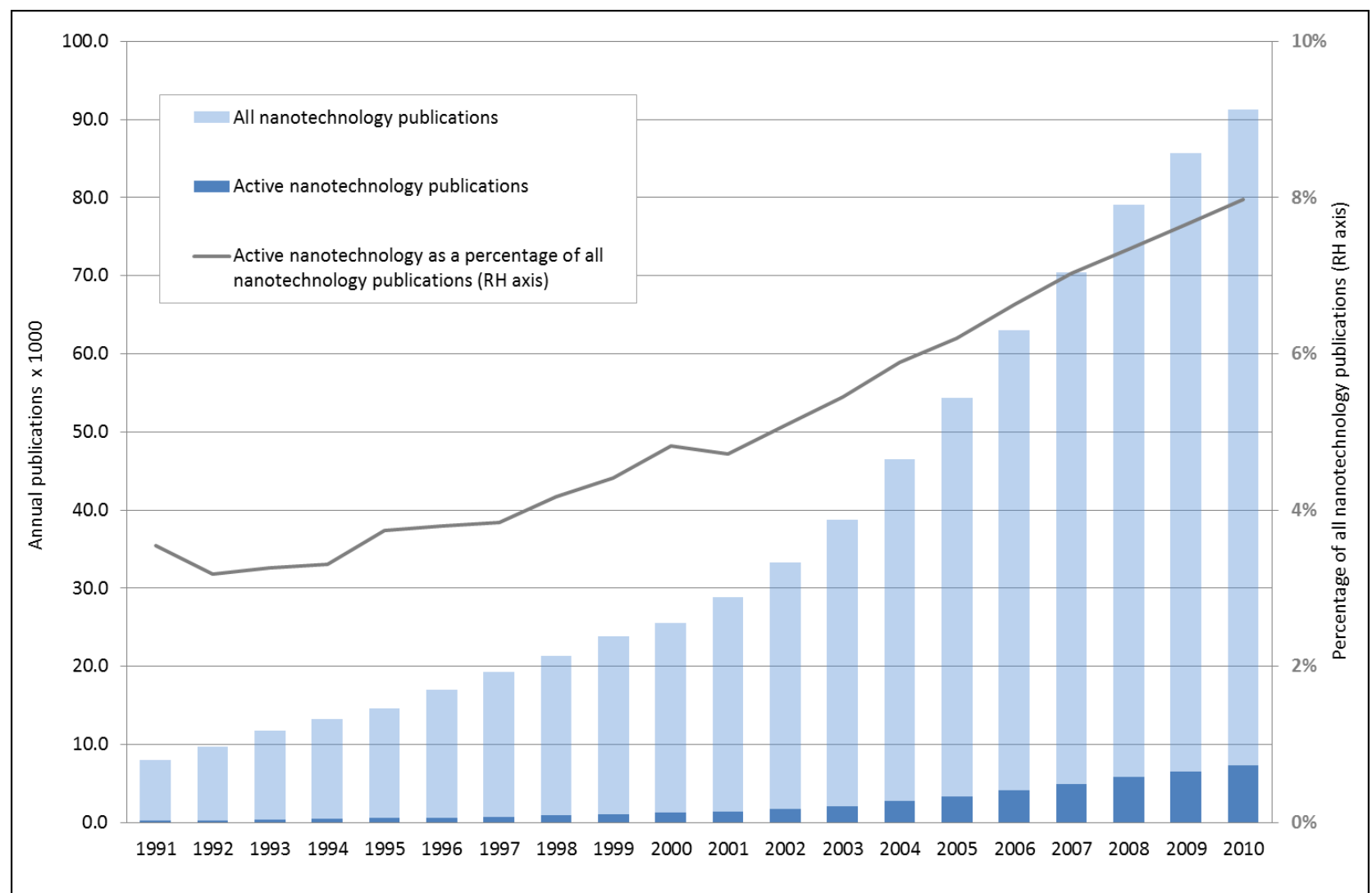

Fig. 3 Growth of active nanotechnology, 1991-2010. Source: Georgia Tech Global Nanotechnology dataset (see text). 


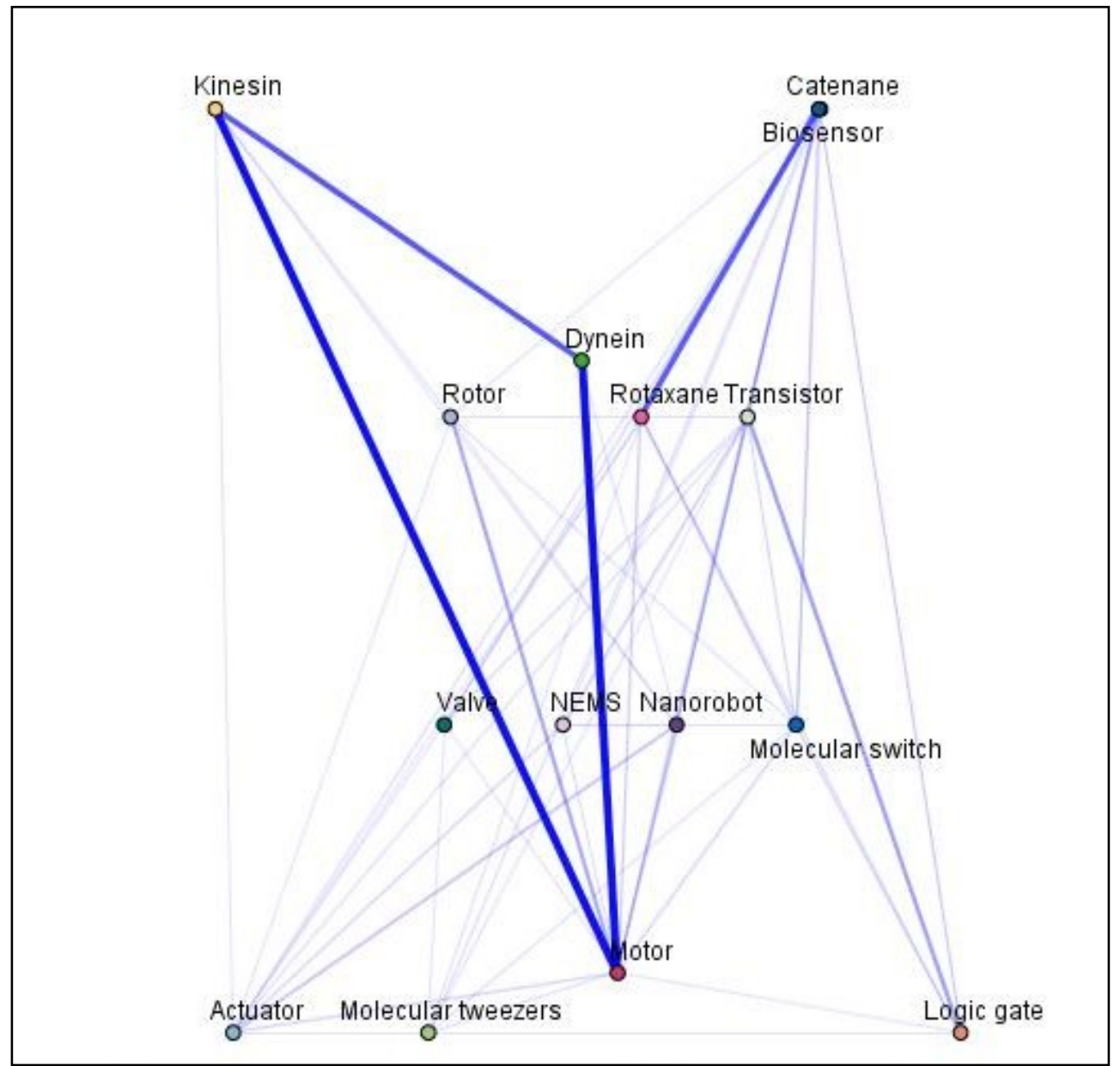

Fig. 4 Keyword co-occurrence network diagram 


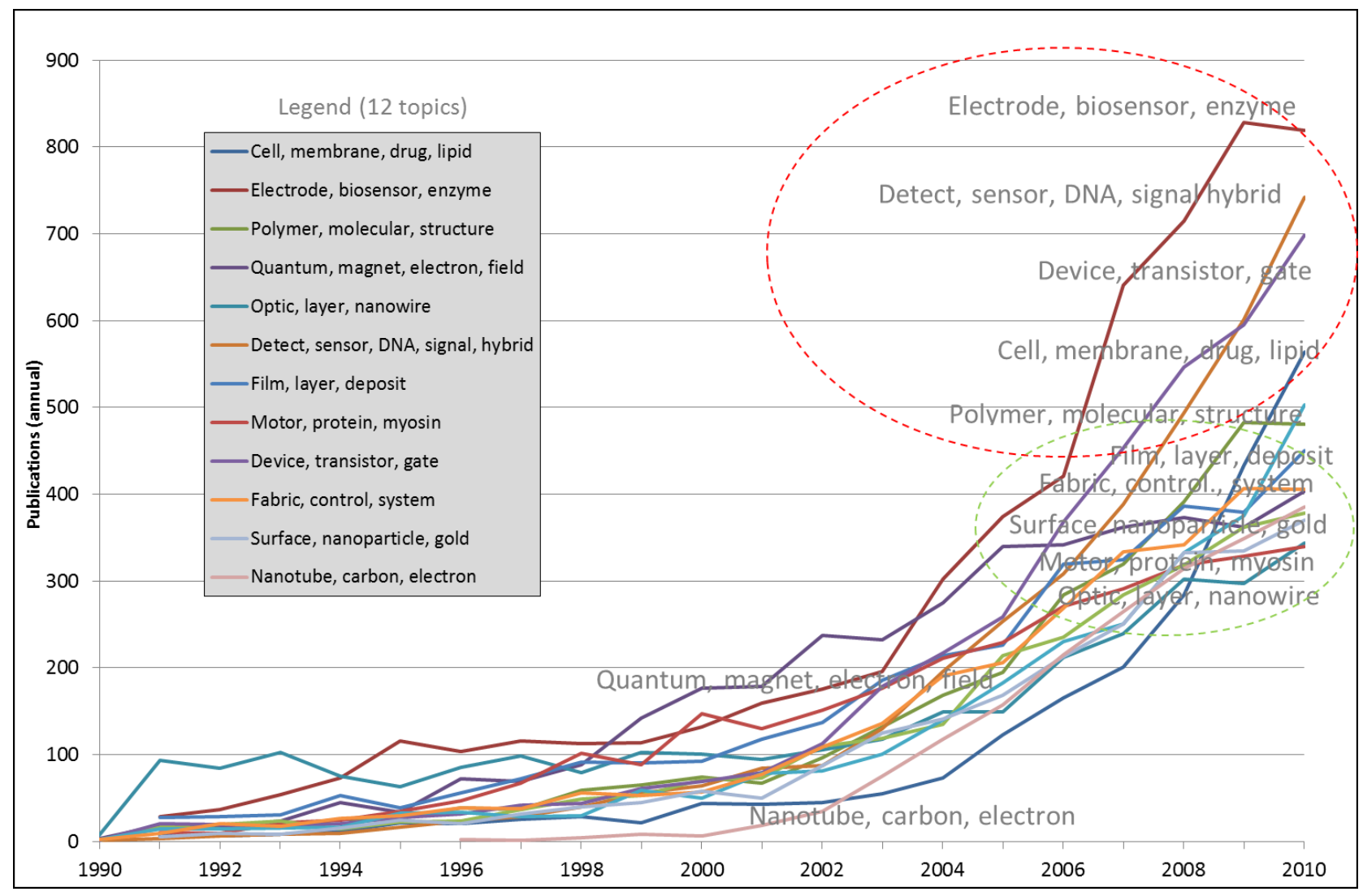

Fig. 5 Active nanotechnology topics, publications by year 
Table 1 Final vocabulary for active nanotechnology

\begin{tabular}{ll}
\hline Type & Terms \\
\hline Activity & biosensing + biosensor/s \\
Functional & molecular switch, motor/s, nanocarrier/s + nanocars, molecular tweezers + molecular clips, \\
parts & actuator/s, rotor, shuttle/s, valve/s, logic gate, transistor, NEMS + nanoelectromechanical system \\
Proper nouns & rotaxane/s, catenane/s, kinesin, dynein \\
Systems & nanorobot / nano robot / robotic nano \\
\hline
\end{tabular}

Note: Terms are included even if they are substrings of other string values. 
Table 2 Number and distribution of engineered nanomaterials in active nanotechnology publications

\begin{tabular}{|c|c|c|c|c|c|c|c|c|c|c|}
\hline \multirow{2}{*}{$\begin{array}{l}\text { Active } \\
\text { nanotechnology } \\
\text { element }\end{array}$} & \multicolumn{9}{|c|}{ Reference to active nanotechnology element - percentage by engineered material type* } & \multirow[t]{2}{*}{ Publications } \\
\hline & gold & $\begin{array}{r}\text { carbon } \\
\text { nanotube }\end{array}$ & silver & $\begin{array}{r}\text { quantum } \\
\text { dot }\end{array}$ & platinum & fullerene & graphene & titanium & dendrimer & \\
\hline transistor & $23 \%$ & $42 \%$ & $5 \%$ & $15 \%$ & $2 \%$ & $11 \%$ & $11 \%$ & $3 \%$ & $0 \%$ & 7,386 \\
\hline biosensor & $67 \%$ & $17 \%$ & $20 \%$ & $2 \%$ & $12 \%$ & $1 \%$ & $1 \%$ & $2 \%$ & $2 \%$ & 2,625 \\
\hline motor & $45 \%$ & $27 \%$ & $13 \%$ & $7 \%$ & $7 \%$ & $8 \%$ & $2 \%$ & $4 \%$ & $0 \%$ & 460 \\
\hline actuator & $24 \%$ & $55 \%$ & $10 \%$ & $1 \%$ & $9 \%$ & $3 \%$ & $3 \%$ & $9 \%$ & $0 \%$ & 331 \\
\hline valve & $26 \%$ & $17 \%$ & $16 \%$ & $11 \%$ & $3 \%$ & $5 \%$ & $14 \%$ & $18 \%$ & $0 \%$ & 255 \\
\hline kinesin & $36 \%$ & $40 \%$ & $6 \%$ & $17 \%$ & $0 \%$ & $9 \%$ & $0 \%$ & $0 \%$ & $0 \%$ & 201 \\
\hline rotaxane & $40 \%$ & $17 \%$ & $8 \%$ & $0 \%$ & $3 \%$ & $30 \%$ & $0 \%$ & $8 \%$ & $6 \%$ & 184 \\
\hline nanocar & $39 \%$ & $10 \%$ & $10 \%$ & $2 \%$ & $6 \%$ & $10 \%$ & $1 \%$ & $1 \%$ & $38 \%$ & 142 \\
\hline$N E M S$ & $24 \%$ & $57 \%$ & $4 \%$ & $8 \%$ & $2 \%$ & $2 \%$ & $11 \%$ & $2 \%$ & $0 \%$ & 98 \\
\hline rotor & $25 \%$ & $13 \%$ & $9 \%$ & $8 \%$ & $7 \%$ & $40 \%$ & $0 \%$ & $5 \%$ & $1 \%$ & 96 \\
\hline logic gate & $21 \%$ & $43 \%$ & $5 \%$ & $19 \%$ & $0 \%$ & $10 \%$ & $4 \%$ & $5 \%$ & $0 \%$ & 90 \\
\hline molecular switch & $64 \%$ & $13 \%$ & $7 \%$ & $2 \%$ & $7 \%$ & $12 \%$ & $1 \%$ & $4 \%$ & $2 \%$ & 89 \\
\hline dynein & $33 \%$ & $11 \%$ & $11 \%$ & $33 \%$ & $0 \%$ & $0 \%$ & $0 \%$ & $11 \%$ & $0 \%$ & 61 \\
\hline catenane & $48 \%$ & $4 \%$ & $12 \%$ & $1 \%$ & $8 \%$ & $34 \%$ & $0 \%$ & $3 \%$ & $3 \%$ & 46 \\
\hline nanorobot & $27 \%$ & $70 \%$ & $6 \%$ & $3 \%$ & $0 \%$ & $3 \%$ & $3 \%$ & $3 \%$ & $0 \%$ & 9 \\
\hline tweezer & $4 \%$ & $0 \%$ & $13 \%$ & $0 \%$ & $9 \%$ & $57 \%$ & $0 \%$ & $0 \%$ & $17 \%$ & 6 \\
\hline Publications & 6,952 & 4,178 & 1,961 & 1,117 & 1,073 & 967 & 824 & 479 & 216 & 11,540 \\
\hline
\end{tabular}

*Percentages sum across; totals may exceed $100 \%$ due to references in a given active nanotechnology publication to more than one engineered nanomaterial. 
Appendix 1 Initial tests of active nanotechnology keywords in nanotechnology titles and abstracts

\begin{tabular}{|c|c|c|c|}
\hline \multirow{2}{*}{$\begin{array}{l}\text { Keywords } \\
\end{array}$} & \multicolumn{2}{|c|}{ Publication counts } & \multirow[t]{2}{*}{ Comments } \\
\hline & title & abstract & \\
\hline & 275 & 718 & not commonly used by scientists \\
\hline motor, rotor & 1,996 & 5,813 & good \\
\hline actuat* & 953 & 3,077 & \\
\hline sens* & 30,181 & 84,279 & includes everything \\
\hline switch, shuttle, smart, responsive & 7,578 & 20,594 & \\
\hline antenna, wireless & 803 & 2,144 & not active terms \\
\hline adaptive & 298 & 1,025 & not an active term \\
\hline memory & 2,670 & 7,358 & does not link to specific active nano technology \\
\hline device & 9,881 & 67,929 & too general \\
\hline transistor & 5,779 & 16,348 & \\
\hline valve & 734 & 2,014 & \\
\hline logic gate & 71 & 252 & \\
\hline self-healing & 118 & 272 & \\
\hline intelligent & 144 & 584 & \\
\hline NEMS & 120 & 587 & \\
\hline nanofluidics & 78 & 239 & \\
\hline systems & 9,069 & 65,998 & too general \\
\hline interface, junction & 11,666 & 63,088 & too general \\
\hline impeller & 6 & 44 & too low counting \\
\hline self-assembling & 1,451 & 4,319 & \\
\hline wire electrode arrays & 1 & 3 & too specific \\
\hline mechanical oscillators & 61 & 80 & \\
\hline self-assembl* \& electronics & 57 & 1,375 & \\
\hline layers & 23,949 & 107,338 & not an active term \\
\hline architecture & 23,94 & 10,771 & too general \\
\hline network & 4,872 & 23,820 & too general \\
\hline direct self assemb1* & 0 & 1 & \\
\hline 3D device & 1 & 15 & \\
\hline dynamic & 20,121 & 84,426 & a generic topic \\
\hline nano + bio & 12,189 & 394,498 & $\begin{array}{l}\text { mostly being captured by terms describing } \\
\text { functional parts }\end{array}$ \\
\hline cage & 1,554 & 6,364 & a nano phenomenon instead of technology \\
\hline bioactive nano & 53 & 93 & \\
\hline nanoelectronics & 327 & 1,366 & a general topic \\
\hline nano-bio interface & 4 & 8 & \\
\hline neuro-prosthesis & 0 & 0 & \\
\hline reactive devices & 0 & 0 & \\
\hline active devices & 22 & 201 & \\
\hline
\end{tabular}




\begin{tabular}{|c|c|c|c|}
\hline neuro-electronic interfaces & 0 & 1 & \\
\hline nanomanufacturing & 59 & 164 & \\
\hline \multicolumn{4}{|l|}{ Third Generation } \\
\hline bio-assembl* & 3 & 6 & \\
\hline nanorobotics & 20 & 99 & \\
\hline regenerative & 135 & 730 & \\
\hline brain-machine interface & 1 & 6 & \\
\hline nanosystems & 363 & 1,076 & a general topic \\
\hline behaviour robotics & 0 & 0 & \\
\hline evolutionary & 156 & 947 & \\
\hline \multicolumn{4}{|l|}{ Fourth Generation } \\
\hline neuromorphic & 16 & 43 & \\
\hline molecular nano & 632 & 1298 & \\
\hline molecular systems & 241 & 1454 & \\
\hline bio+assembly & & & $\begin{array}{l}\text { issues in separating bio-related assembly process } \\
\text { from chemical process }\end{array}$ \\
\hline tissue engineering & & & a generic topic \\
\hline post-CMOS & & & no specific technology been studied; too few counts \\
\hline suface+robot & & & too few counts \\
\hline $\mathrm{bio}+$ nano & & 39,4498 & captured by terms describing functional parts \\
\hline
\end{tabular}

Note: Test applied to Georgia Tech Global Nanotechnology dataset (see text). The '*' is a wildcard symbol that represents multiple variations of the term 